Modèles linguistiques

ling uistiques

$3 \mid 2010$

Jean-Claude Chevalier. Chroniques de linguistique dans La Quinzaine Littéraire (1975-2010)

\title{
La parole intérieure
}

Jean-Claude Chevalier

\section{OpenEdition}

Journals

Édition électronique

URL : https://journals.openedition.org/ml/427

DOI : $10.4000 / \mathrm{ml} .427$

ISSN : 2274-0511

Éditeur

Association Modèles linguistiques

Édition imprimée

Date de publication : 1 décembre 2010

Pagination : 123-126

Référence électronique

Jean-Claude Chevalier, «La parole intérieure », Modèles linguistiques [En ligne], 3 | 2010, mis en ligne le 23 octobre 2013, consulté le 01 juillet 2021. URL : http://journals.openedition.org/ml/427 ; DOI :

https://doi.org/10.4000/ml.427

Ce document a été généré automatiquement le 1 juillet 2021

(C) Modèles Linguistiques 


\title{
La parole intérieure
}

\author{
Jean-Claude Chevalier
}

$\mathrm{N}^{\circ} 878,1-15$ juin 2004

Gabriel BERGOUNIOUX

Le moyen de parler

Verdier éd., 238 p.

\begin{abstract}
Le titre même est un paradoxe, car il ne s'agit pas ici de " parler », au sens ordinaire du mot, mais principalement de cette parole muette qu'est la parole intérieure, le monologue intérieur, enchâssé par les écrivains, de Dujardin à Joyce et O'Neill, et bien d'autres, qui est notre compagnie ordinaire par la prière ou les soliloques; ou en toute autre occasion et elle sont multiples - de rumination.
\end{abstract}

1 Tenter de comprendre en savant ce phénomène de la parole intérieure, de l'endophasie comme on dit, c'est affronter les problèmes centraux de la linguistique en privilégiant l'écoute. Ce que fait Gabriel Bergounioux en linguiste compétent qu'il est - un des meilleurs aujourd'hui - et aussi un lecteur attentif de Bourdieu; comme tel, il substitue à l'attendue problématique du sujet un centrage sur l'agent en même temps qu'il questionne la nature de l'objet « langue ». En ce sens, l'« homme de parole » n'est ni actif ni passif, il est un lieu intermédiaire d'échanges. Et en fait de parole, il y a surtout ici une écoute intérieure.

Difficulté à saisir les manifestations de l'endophasie qui échappe aux traitements expérimentaux du dire. La grammaire est d'abord, et depuis l'origine, une grammaire de l'écrit qui joue des échanges entre la trame sonore et l'histoire gravée dans l'archive. L'étude des aphasies au XIX ${ }^{\mathrm{e}} \mathrm{s}$. (par Broca, en premier) introduit pourtant à la parole intérieure. Plus tard, la phonétique et ses transcriptions donnent encore plus de souplesse à l'approche du linguiste. Mais Saussure, puis Chomsky, entre autres, isolent «la langue » de ses agents. La parole apparait comme un résidu. En témoigne, chez 
Bergounioux, l'analyse de "Pauv'con », «L'autre », etc. Alors, et je le cite, l'endophasie est un enjeu fort; ; elle représente l'envers non formalisable du projet scientifique de la linguistique ».

3 Après l'étude du «dire" se pose le problème de l'« entendre ", éclairé par les phonéticiens. Le discours intérieur situe le parleur, la communauté qu'il vise, les informations qu'il recherche, les structures qu'il aime identifier. D'où le paradoxe: «Entendre, c'est avoir à (re)connaître ce qui (nous) est dit par ce qui se dit et ce qu'on se dit, c'est se rappeler». La mémoire est donc «la modalité fondatrice de son fonctionnement». Elle est un processus actif et créatif. Et c'est là que le discours intérieur joue un rôle décisif : il reconstruit le souvenir des discours passés; il est action et énergie. Il alterne avec l'activité extérieure, tangible et interpersonnelle, marquée dans son discours propre. Du conflit résultent concurrences, conférences, interférences.

4 Se replier sur l'un ou l'autre discours suppose un coupe-circuit. On "décroche ", ou plutôt, on masque l'autre discours. Ce qu'exhibent le bavardage amoureux ou le babillage brillant du conférencier. Dont le répondant intérieur se déploie librement dans la songerie ou dans les rêves mêmes, libre des exigences de l'interaction, abandonné aux coq-à-l'âne de la condensation et du déplacement. Phénomène psychique, dominant, écrasant, grâce à l'économie de l'articulation, les bornes de discours précuits, si l'on peut dire. La parole interne radote et rabâche ; remembrances qu'on peut inventorier, rebondissant sur des homonymies, strictes ou approximatives, comme houppe, août, ouf et housse. Et autres mécanismes homologues : comme cette union des contraintes contenue dans " chasser » ("pousser devant soi" ou "chercher à prendre"), telle que l'ont commentée Freud et Benveniste.

5 Endophasies de tous types, stimulées par les acousmates ou les hallucinations auditives, qui construisent notre univers profond avec ses interlocuteurs privilégiés : l'être aimé ou haï, la mère, en particulier, dit-il. Alors « l'ordre du monde est inséparable de l'ordre du discours ».

6 Cela dit, les phonéticiens notent que tout auditeur est polylectal (Rousselot, Gillieron). A l'inverse, les grammaires contemporaines, comme le chomskysme, ont ramené le vivant à l'ordre logique de l'électronique. Ce qui le distancie de l'ordre intérieur fondé sur les enchâssements et les enchaînements. Car le discours est engendré par le discours (le "Ça parle» de Lacan); il est une pratique sociale. Ici, on touche le point nodal. Toutes les opérations du psychisme relèvent de la langue. Comme l'inconscient de Lacan, la conscience, le moi, le sujet sont référés au langage. Par l'énonciation, grâce à son appareil formel (Benveniste), le moi s'inscrit dans la langue - à moins que le locuteur ne soit l'effet des marques énoncées. "L'usage de la langue est moins requis pour dire le monde qu'elle n'est la façon de le produire ». Mais, paradoxe difficile à réduire, déploiement du discours et fonctionnement de la pensée ne coïncident qu'imparfaitement.

7 Le lieu de contact, l'instrument unificateur, c'est la " quote », séquence conforme aux principes de la langue sans en avoir les propriétés; «à la fois cause et configuration de l'explication infinie de l'auditeur à lui-même ", elle concentre en une suite restreinte de phonèmes un discours. Image jamais achevée, soumise à versatilité et complainte aux captations de l'émetteur ; organisée pourtant; ni holophrase, ni nœud. « La quote est potentielle ", dit Bergounioux; et c'est un peu fuyant. On y référera le style du stream of consciousness des romans anglais, les termes de la psychanalyse (par 
exemple, les trois formes valorisées par Freud dans la deuxième topique : moi, ça, surmoi), ceux de la sociologie même. Si l'on était tenté au début de se reporter aux analyses des maitres en psychologie de l'autre siècle comme Th. Ribot, on verrait que l'auteur même interdit clairement un tel rapprochement.

8 À partir de là, le lecteur doit prendre du champ. L'existence de la "quote " pose la question: «Qu'est-ce que comprendre? » La psychanalyse a mis en vogue l'écoute. L'auteur précise: comprendre, c'est accueillir la version de l'interlocuteur et c'est surtout la situer dans les innombrables passages entre les groupes sociaux. Et donc la diviser, la déchirer. À l'infini. Encore qu'il y ait un « temps pour comprendre ", comme dit Lacan, qui peut recouvrir un jour aussi bien qu'une vie entière, un temps pour résoudre des discours contradictoires. Que parasitent les actes manqués et l'endophasie. Dispositif que l'auteur interprète, sur un mode marxiste, en l'inscrivant dans un ensemble de rapports sociaux; et en situant là l'endophasie qui ferait de la pensée un dialogue à la recherche obstinée de la compréhension.

9 Ce dire silencieux est proche du rêve. Et de l'être-là le plus profond de l'individu. Je cite :

Dans la forme particulière où est caractérisée une espèce (homo sapiens sapiens) et la nécessité de sa réalisation, ce qu'énonce l'endophasie se confond avec l'essence de l'homme en tant que l'une, l'essence, et l'autre, la parole lui sont extrinsèques et qu'il ne croit s'y entendre que parce qu'elles le précèdent et le comprennent, socialement, linguistiquement.

10 Un «Dernier mot», surprenant, de deux pages. L'auteur, dont le manuscrit entier repose sur l'intuition, réclame maintenant, à cor et à cri, des preuves empiriques. Mais que peut faire un linguiste d'une notion à laquelle ne répond aucun corpus ferme ? Au mieux, par flair, déceler le discours latent de l'endophasie dans la superposition des codes. Entreprise scientifique ou humour ravageur porté sur la condition du linguiste, c'est au lecteur à choisir.

11 Livre captivant, étrange, difficile parfois, profondément original dans le champ linguistique actuel. Qui organise un sujet neuf, ou du moins malmené, dans les filets d'une vaste culture. Publication d'autant plus saillante qu'elle s'est doublée, au même moment, d'une production romanesque saisissante, dès son titre même: Il y a un ${ }^{1}$. Comme une "quote" qui évoque la présence lancinante de l'indéfini, récit phénoménologique qu'on peut faire remonter à Camus. Linéaire et sans fin, mettant en scène un personnage affrontant la guerre et les violences et continuant malgré tout une existence imprévisible. Un réalisme fantastique traversé de rage rentrée. Autre aspect de l'« homme du parler et de l'écriture déchiqueté par l'angoisse de vivre ».

\section{NOTES}

1. Gabriel Bergounioux, Il y a un, roman aux éditions Champvallon, Collection Détours, 2004. 247 pages.

Gabriel Bergounioux, éditeur de La parole intérieure, "Langue française » 132, décembre 2001, Larousse, Paris. 